\title{
SPECTROPHOTOMETRICAL MODELS FOR AGN
}

\author{
DORU MARIAN SURAN and NEDELIA ANTONIA POPESCU \\ Astronomical Institute of the Romanian Academy, \\ 75212 Bucharest 28 ROMANIA
}

\begin{abstract}
We compare the observational colour diagrams with the position of the lensed quasars,mean cluster memberships for $z<1$ and galactic evolutive tracks in order to determine the influence of these effects in AGN evolution.
\end{abstract}

\section{OBSERVATIONAL DATA}

We have used the following catalogs: Catalog of Quasars and Active Nuclei (Veron Cetty, Veron 1991); Catalog of lensed quasars (Suran, Popescu 1992); Mean evolutive colours for galaxies in high redshift clusters (Suran, Popescu 1987); Atlas of synthetic spectra of galaxies (Rocca-Volmerange, Guideroni 1988).

\section{RESULTS AND CONCLUSIONS}

The comparison between the different data in the two colour diagrams (U-B, B-V) and in the cosmological diagrams $(\mathrm{U}-\mathrm{B}, \mathrm{z}),(\mathrm{B}-\mathrm{V}, \mathrm{z})$ reveals three distinct regions: AGN with $z<0.8-1.2$; quasars with $0.8-1.2<z<2.5-3$; quasars with $z>2.5-3$. Also, different effects as lensing, cluster and cosmological effects have been discussed.

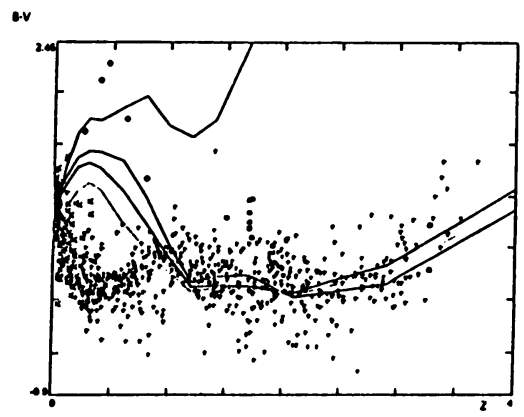

Fig. 1. The cosmological diagram (B-V,z). (+): quasars, (x): Seyfert gal., (squares): lensed quasars, (diamonds): clusters, lines: differents tracks of galactic evolution from burst to Im (Rocca-Volmerange and Guideroni 1988).

\section{References}

Rocca-Volmerange,B., Guideroni,B. 1988 Astr.Astrophys.Suppl.Ser.,75,93;

Suran,M.D., Popescu,N.A. 1987, Internal CSEN Report No. 9047;

Suran,M.D., Popescu,C.C. 1992,Rom.Astron.J.,2,1;

Veron-Cetty,M.P., Veron,P. 1991, ESO Report,No.10.

T. J.-L. Courvoisier and A. Blecha: Multi-Wavelength Continuum Emission of AGN, 524.

(C) 1994 IAU. Printed in the Netherlands. 